\title{
Selected Topics in Teleparallel Gravity
}

\author{
R. Aldrovandi, J. G. Pereira, and K. H. Vu \\ Instituto de Física Teórica, Universidade Estadual Paulista \\ Rua Pamplona 145, 01405-900, São Paulo, SP, Brazil
}

Received on 16 January, 2004

\begin{abstract}
Teleparallel gravity can be seen as a gauge theory for the translation group. As such, its fundamental field is neither the tetrad nor the metric, but a gauge potential assuming values in the Lie algebra of the translation group. This gauge character makes of teleparallel gravity, despite its equivalence to general relativity, a rather peculiar theory. A first important point is that it does not rely on the universality of free fall, and consequently does not require the equivalence principle to describe the gravitational interaction. Another peculiarity is its similarity with Maxwell's theory, which allows an Abelian nonintegrable phase factor approach, and consequently a global formulation for gravitation. Application of these concepts to the motion of spinless particles, as well as to the COW and gravitational Aharonov-Bohm effects are presented and discussed.
\end{abstract}

\section{Introduction}

Like the other fundamental interactions of nature, gravitation can be described by a gauge theory [1]. The teleparallel equivalent of general relativity [2], or teleparallel gravity for short [3], can indeed be understood as a gauge theory for the translation group. In this approach, the gravitational interaction is described by a force similar to the Lorentz force equation of electrodynamics, with torsion playing the role of force [4].

On the other hand, due to the universality of free fall, it is also possible to describe gravitation not as a force, but as a geometric deformation of flat Minkowski spacetime. According to this point of view, a gravitational field produces a curvature in spacetime, and its action on (structureless) particles is described by letting them follow the geodesics of the curved spacetime. This is the approach of general relativity, in which geometry replaces the concept of gravitational force, and the trajectories are determined, not by force equations, but by geodesics. We notice in passing that an immediate consequence of this dual description of gravitation is that curvature and torsion might be related with the same degrees of freedom of the gravitational field.

As a gauge theory for the translation group, which is an Abelian group, the teleparallel formulation of gravity becomes in several aspects similar to the electromagnetic Maxwell's theory. By exploring this analogy, as well as by using well known results of electrodynamics, the basic purpose of this paper will be to study some specific properties of teleparallel gravity.

The first point to be examined refers to the weak equivalence principle, which establishes the equality of inertial and gravitational masses. As is widely known, the electromagnetic interaction is not universal and there exists no electromagnetic equivalence principle. Nevertheless, Maxwell's theory, a gauge theory for the Abelian group $U(1)$, descri- bes quite consistently the electromagnetic interaction. Given the analogy between electromagnetism and teleparallel gravity, in which the equations of motion are not geodesics but force equations quite analogous to the electromagnetic Lorentz force equation, the question then arises whether the gauge approach of teleparallel gravity would also be able to describe the gravitational interaction in the lack of universality, that is, in the absence of the weak equivalence principle. As we are going to see, the answer to this question is positive: teleparallel gravity does not require the validity of the equivalence principle to describe the gravitational interaction. In fact, although the geometrical description of general relativity breaks down, the gauge description of teleparallel gravity remains as a consistent theory in the absence of universality [5]. It belongs, therefore, to a more general type of theory.

A second point to be explored is the so called global formulation, which is an approach based on the action of a nonintegrable phase factor. Relying on the well known phase factor approach to Maxwell's theory [6], a teleparallel nonintegrable phase factor approach to gravitation will be developed, which represents the quantum mechanical version of the classical gravitational Lorentz force of teleparallel gravity. As a first application of this global approach, we consider the Colella, Overhauser, Werner (COW) experiment [7], which consists in using a neutron interferometer to observe the quantum mechanical phase shift of neutrons caused by their interaction with Earth's gravitational field. By considering the Newtonian limit, it is shown that the teleparallel global formalism yields the correct quantum phaseshift predicted (as well as experimentally verified) for the COW experiment. As a second application of the teleparallel global approach, we obtain the quantum phase-shift produced by the coupling of the particle's kinetic energy with the gravitomagnetic components of the translational gauge potential [8]. This effect is the gravitational analog of the 
usual electromagnetic Aharonov-Bohm effect, and for this reason it will be called the gravitational Aharonov-Bohm effect [9]. It is worthy mentioning that, as the phase difference depends essentially on the energy, it applies equally to massive and massless particles. For the sake of completeness, we begin by reviewing, in the next section, the basic concepts related to teleparallel gravity. The equivalence principle is recast in its language, and shown to be unnecessary. The global approach to gravitation is then presented and applied to the two mentioned effects.

\section{Fundamentals of Teleparallel Gra- vity}

Teleparallel gravity corresponds to a gauge theory of the translation group. According to this model, to each point of spacetime there is attached a Minkowski tangent space, on which the translation (gauge) group acts. We use the Greek alphabet $\mu, \nu, \rho, \cdots=0,1,2,3$ to denote spacetime indices and the Latin alphabet $a, b, c, \cdots=0,1,2,3$ to denote anholonomic indices related to the tangent Minkowski spaces, whose metric is chosen to be $\eta_{a b}=\operatorname{diag}(+1,-1,-1,-1)$. As a gauge theory for translations, the fundamental field of teleparallel gravity is the translational gauge potential $B^{a}{ }_{\mu}$, a 1-form assuming values in the Lie algebra of the translation group:

$$
B_{\mu}=B^{a}{ }_{\mu} P_{a}
$$

with $P_{a}=\partial_{a}$ the generators of infinitesimal translations. Under a local translation of the tangent space coordinates $\delta x^{a}=\epsilon^{a}(x)$, the gauge potential transforms according to

$$
B^{\prime a}{ }_{\mu}=B^{a}{ }_{\mu}-\partial_{\mu} \epsilon^{a} .
$$

It appears naturally as the nontrivial part of the tetrad field $h_{\mu}^{a}$ :

$$
h^{a}{ }_{\mu}=\partial_{\mu} x^{a}+B^{a}{ }_{\mu} .
$$

Notice that, whereas the tangent space indices are raised and lowered with the Minkowski metric $\eta_{a b}$, the spacetime indices are raised and lowered with the spacetime metric

$$
g_{\mu \nu}=\eta_{a b} h_{\mu}^{a} h_{\nu}^{b} .
$$

The above tetrad gives rise to the so called Weitzenböck connection

$$
\Gamma_{\mu \nu}^{\rho}=h_{a}{ }^{\rho} \partial_{\nu} h^{a}{ }_{\mu},
$$

which introduces the distant parallelism in the fourdimensional spacetime manifold. It is a connection which presents torsion, but no curvature. Its torsion,

$$
T_{\mu \nu}^{\rho}=\Gamma_{\nu \mu}^{\rho}-\Gamma_{\mu \nu}^{\rho},
$$

is related to the translational gauge field strength $F^{a}{ }_{\mu \nu}$ by

$$
F^{a}{ }_{\mu \nu} \equiv \partial_{\mu} B_{\nu}^{a}-\partial_{\nu} B_{\mu}^{a}=h_{\rho}^{a} T_{\mu \nu}^{\rho} .
$$

The Weitzenböck connection can be decomposed as

$$
\Gamma_{\mu \nu}^{\rho}=\stackrel{\circ}{\Gamma}_{\mu \nu}+K_{\mu \nu}^{\rho},
$$

where $\stackrel{\circ}{\Gamma}_{\mu \nu}{ }_{\mu \nu}$ is the Christoffel connection constructed from the spacetime metric $g_{\mu \nu}$, and

$$
K_{\mu \nu}^{\rho}=\frac{1}{2}\left(T_{\mu}{ }^{\rho}{ }_{\nu}+T_{\nu}{ }^{\rho}{ }_{\mu}-T_{\mu \nu}^{\rho}\right)
$$

is the contortion tensor. It is important to remark that curvature and torsion are considered as properties of a connection, not of spacetime [10]. Notice, for example, that the Christoffel and the Weitzenböck connections are defined on the very same spacetime manifold.

The Lagrangian of the teleparallel equivalent of general relativity is [4]

$$
\mathcal{L} \equiv \mathcal{L}_{G}+\mathcal{L}_{M}=\frac{c^{4} h}{16 \pi G} S^{\rho \mu \nu} T_{\rho \mu \nu}+\mathcal{L}_{M}
$$

where $h=\operatorname{det}\left(h^{a}{ }_{\mu}\right), \mathcal{L}_{M}$ is the Lagrangian of a source field, and

$$
S^{\rho \mu \nu}=-S^{\rho \nu \mu}=\frac{1}{2}\left[K^{\mu \nu \rho}-g^{\rho \nu} T_{\sigma}^{\sigma \mu}+g^{\rho \mu} T_{\sigma}^{\sigma \nu}\right]
$$

is a tensor written in terms of the Weitzenböck connection only. Performing a variation with respect to the gauge potential, we find the teleparallel version of the gravitational field equation [11],

$$
\partial_{\sigma}\left(h S_{\lambda}^{\rho \sigma}\right)-\frac{4 \pi G}{c^{4}}\left(h t_{\lambda}^{\rho}\right)=\frac{4 \pi G}{c^{4}}\left(h \mathcal{T}_{\lambda}{ }^{\rho}\right)
$$

where

$$
h t_{\lambda}{ }^{\rho}=\frac{c^{4} h}{4 \pi G} S_{\mu}{ }^{\rho \nu} \Gamma^{\mu \lambda}-\delta_{\lambda}{ }^{\rho} \mathcal{L}_{G}
$$

is the energy-momentum pseudotensor of the gravitational field, and $\mathcal{T}_{\lambda}{ }^{\rho}=\mathcal{T}_{a}{ }^{\rho} h^{a}{ }_{\lambda}$ is the energy-momentum tensor of the source field, with

$$
h \mathcal{T}_{a}{ }^{\rho}=-\frac{\delta \mathcal{L}_{M}}{\delta B^{a}{ }_{\rho}} \equiv-\frac{\delta \mathcal{L}_{M}}{\delta h^{a}{ }_{\rho}} .
$$

A solution of the gravitational field equation (12) is an explicit form of the gravitational gauge potential $B^{a}{ }_{\mu}$.

When the weak equivalence principle is assumed to be true, teleparallel gravity turns out to be equivalent to general relativity. In fact, up to a divergence, the Lagrangian (10) is found to be equivalent to the Einstein-Hilbert Lagrangian of general relativity, and the teleparallel field equation (12) is found to coincide with Einstein's equation

$$
\stackrel{\circ}{R}_{\lambda}^{\rho}-\frac{1}{2} \delta_{\lambda}{ }^{\rho} \stackrel{\circ}{R}=\frac{8 \pi G}{c^{4}} \mathcal{T}_{\lambda}^{\rho}
$$

with $\stackrel{\circ}{R}_{\lambda}^{\rho}$ and $\stackrel{\circ}{R}$ respectively the Ricci and the scalar curvature of the Christoffel connection. 


\section{Gravitation and the Weak Equiva- lence Principle}

Let us begin by making it clear that, in spite of many controversies related with the equivalence principle [12], it is not our intention here to question its validity, but simply to verify whether teleparallel gravity requires it or not to describe the gravitational interaction. This will be done by supposing that the gravitational mass $m_{g}$ and the inertial mass $m_{i}$ do not coincide, and then by making a comparative study of the force equation of teleparallel gravity with the geodesic equation of general relativity.

\subsection{Teleparallel Gravity: Force Equation}

Let us then consider, in the context of teleparallel gravity, the motion of a spinless particle in a gravitational field $B^{a}{ }_{\mu}$, supposing however that the gravitational and the inertial masses do not coincide. Analogously to the electromagnetic case [13], the action integral is written in the form

$$
S=\int_{a}^{b}\left[-m_{i} c d \sigma-m_{g} c B_{\mu}^{a} u_{a} d x^{\mu}\right],
$$

where $d \sigma=\left(\eta_{a b} d x^{a} d x^{b}\right)^{1 / 2}$ is the Minkowski tangentspace invariant interval, and $u^{a}$ is the particle four-velocity seen from the tetrad frame, necessarily anholonomic when expressed in terms of the spacetime line element $d s$. The first term of the action (16) represents the action of a free particle, and the second the (minimal) coupling of the particle with the gravitational field. Variation of the action (16) yields the equation of motion [5]

$$
\left(\partial_{\mu} x^{a}+\frac{m_{g}}{m_{i}} B^{a}{ }_{\mu}\right) \frac{d u_{a}}{d s}=\frac{m_{g}}{m_{i}} F^{a}{ }_{\mu \rho} u_{a} u^{\rho},
$$

where $F^{a}{ }_{\mu \rho}$ is the gravitational field strength defined in Eq. (7), and

$$
u^{\mu}=\frac{d x^{\mu}}{d s} \equiv h^{\mu}{ }_{a} u^{a}
$$

is the holonomic four-velocity, with $d s=\left(g_{\mu \nu} d x^{\mu} d x^{\nu}\right)^{1 / 2}$ the Riemannian spacetime invariant interval. Equation (17) is the force equation governing the motion of the particle, in which the teleparallel field strength $F^{a}{ }_{\mu \rho}$ (that is, the Weitzenböck torsion) plays the role of gravitational force. Similarly to the electromagnetic Lorentz force, which depends on the relation $e / m_{i}$, with $e$ the electric charge of the particle, the gravitational force depends explicitly on the relation $m_{g} / m_{i}$ of the particle.

We see from the above equations that, even in the absence of the weak equivalence principle, teleparallel gravity is able to describe the motion of a particle with $m_{g} \neq m_{i}$. The crucial point is to observe that, although the equation of motion depends explicitly on the relation $m_{g} / m_{i}$ of the particle, neither $B^{a}{ }_{\mu}$ nor $F^{a}{ }_{\rho \mu}$ depends on this relation. This means essentially that the teleparallel field equation (12) can be consistently solved for the gravitational potential $B^{a}{ }_{\mu}$, which can then be used to write down the equation of motion (17), independently of the validity or not of the weak equivalence principle. The gauge potential $B^{a}{ }_{\mu}$, therefore, may be considered as the most fundamental field representing gravitation. As we are going to see next, this is not the case of general relativity, in which to keep the equations of motion given by geodesics, the gravitational field (metric tensor) must necessarily depend on the relation $m_{g} / m_{i}$ of the particle, rendering thus the theory inconsistent when $m_{g} \neq m_{i}$.

\subsection{General Relativity: Geodesics}

According to teleparallel gravity, even when $m_{g} \neq m_{i}$, the tetrad is still given by (3), and the spacetime indices are raised and lowered with the metric (4). Then, by using the relation (7), as well as the identity

$$
T_{\mu \rho}^{\lambda} u_{\lambda} u^{\rho}=-K_{\mu \rho}^{\lambda} u_{\lambda} u^{\rho},
$$

the force equation (17) can be rewritten in the form

$$
\frac{d u_{\mu}}{d s}-\stackrel{\circ}{\Gamma}_{\mu \rho}^{\lambda} u_{\lambda} u^{\rho}=\left(\frac{m_{g}-m_{i}}{m_{g}}\right) \partial_{\mu} x^{a} \frac{d u_{a}}{d s},
$$

where use has been made also of the relation (8). Notice that the violation of the weak equivalence principle produces a deviation from the geodesic motion, which is proportional to the difference between the gravitational and inertial masses. Notice furthermore that, due to the assumed nonuniversality of free fall, it is not possible to find a local coordinate system in which the gravitational effects are absent.

Now, as already said, when the weak equivalence principle is assumed to be true, the teleparallel field equation (12) is equivalent to Einstein's equation (15). Accordingly, when $m_{g}=m_{i}$, the equation of motion (17) reduces to the geodesic equation of general relativity, as can be seen from its equivalent form (20). However, in the absence of the weak equivalence principle, it is not a geodesic equation. This means that the equation of motion (17) does not comply with the geometric description of general relativity, according to which all trajectories must be given by genuine geodesic equations. In order to comply with the foundations of general relativity, it is necessary to incorporate the particle properties into the geometry. This can be achieved by assuming, instead of the tetrad (3) of teleparallel gravity, the new tetrad

$$
\bar{h}_{\mu}^{a}=\partial_{\mu} x^{a}+\frac{m_{g}}{m_{i}} B^{a}{ }_{\mu}
$$

which takes into account the characteristic $m_{g} / m_{i}$ of the particle under consideration. This tetrad defines a new spacetime metric tensor

$$
\bar{g}_{\mu \nu}=\eta_{a b} \bar{h}_{\mu}^{a} \bar{h}_{\nu}^{b}
$$

in terms of which the corresponding spacetime invariant interval is

$$
d \bar{s}^{2}=\bar{g}_{\mu \nu} d x^{\mu} d x^{\nu}
$$

By noticing that in this case the relation between the gravitational field strength and torsion becomes

$$
\frac{m_{g}}{m_{i}} F_{\mu \rho}^{a}=\bar{h}_{\lambda}^{a} \bar{T}_{\mu \rho}^{\lambda},
$$


it is an easy task to verify that, for a fixed relation $m_{g} / m_{i}$, the equation of motion (17) is equivalent to the true geodesic equation

$$
\frac{d \bar{u}_{\mu}}{d \bar{s}}-\bar{\Gamma}_{\mu \rho}^{\lambda} \bar{u}_{\lambda} \bar{u}^{\rho}=0,
$$

where $\bar{u}_{\mu} \equiv d x_{\mu} / d \bar{s}=\bar{h}^{a}{ }_{\mu} u_{a}$, and $\bar{\Gamma}^{\rho}{ }_{\mu \nu}$ is the Christoffel connection of the metric $\bar{g}_{\mu \nu}$. However, the price for imposing a geodesic equation of motion to describe a nonuniversal interaction is that the gravitational theory becomes inconsistent. In fact, the solution of the corresponding Einstein's field equation

$$
\bar{R}_{\mu \nu}-\frac{1}{2} \bar{g}_{\mu \nu} \bar{R}=\frac{8 \pi G}{c^{4}} \overline{\mathcal{T}}_{\mu \nu},
$$

which is not equivalent to any teleparallel field equation, would in this case depend on the relation $m_{g} / m_{i}$ of the test particle, which renders the theory inconsistent in the sense that test particles with different relations $m_{g} / m_{i}$ would require connections with different curvatures to keep all equations of motion given by geodesics. Of course, as a true field, the gravitational field cannot depend on any test particle properties.

\section{Global Formulation of Gravitation}

The basic conclusion of the previous section is that the fundamental field describing gravitation is neither the tetrad nor the metric, but the translational gauge potential $B^{a}{ }_{\mu}$. Using this fact, and the similarity of teleparallel gravity with Maxwell's theory, we are going to introduce now a teleparallel nonintegrable phase factor, in terms of which a global formulation for gravitation will be developed.

\subsection{Nonintegrable Phase Factor}

As is well known, in addition to the usual differential formalism, electromagnetism presents also a global formulation in terms of a nonintegrable phase factor [6]. According to this approach, electromagnetism can be considered as the gauge invariant effect of a nonintegrable (path-dependent) phase factor. For a particle with electric charge $e$ traveling from an initial point $\mathrm{P}$ to a final point $\mathrm{Q}$, the phase factor is given by

$$
\Phi_{e}(\mathrm{P} \mid \mathrm{Q})=\exp \left[\frac{i e}{\hbar c} \int_{\mathrm{P}}^{\mathrm{Q}} A_{\mu} d x^{\mu}\right],
$$

where $A_{\mu}$ is the electromagnetic gauge potential. In the classical (non-quantum) limit, the action of this nonintegrable phase factor on a particle wave-function yields the same results as those obtained from the Lorentz force equation

$$
\frac{d u^{a}}{d s}=\frac{e}{m_{i} c^{2}} F_{b}^{a} u^{b} .
$$

In this sense, the phase-factor approach can be considered as the quantum generalization of the classical Lorentz force equation. It is actually more general, as it can be used both on simply-connected and on multiply-connected domains. Its use is mandatory, for example, to describe the Aharonov-Bohm effect, a quantum phenomenon taking place in a multiply-connected space [14].

Now, in the teleparallel approach to gravitation, the fundamental field describing gravitation is the translational gauge potential $B^{a}{ }_{\mu}$. Like $A_{\mu}$, it is an Abelian gauge potential. Thus, in analogy with electromagnetism, $B^{a}{ }_{\mu}$ can be used to construct a global formulation for gravitation. To start with, let us notice that the electromagnetic phase factor $\Phi_{e}(\mathrm{P} \mid \mathrm{Q})$ is of the form

$$
\Phi_{e}(\mathrm{P} \mid \mathrm{Q})=\exp \left[\frac{i}{\hbar} S_{e}\right]
$$

where $S_{e}$ is the action integral describing the interaction of the charged particle with the electromagnetic field. Now, in teleparallel gravity, the action integral describing the interaction of a particle of mass $m_{g}$ with gravitation, according to Eq. (16), is given by

$$
S_{g}=\int_{\mathrm{P}}^{\mathrm{Q}} m_{g} c B^{a}{ }_{\mu} u_{a} d x^{\mu} .
$$

Therefore, the corresponding gravitational nonintegrable phase factor turns out to be

$$
\Phi_{g}(\mathrm{P} \mid \mathrm{Q})=\exp \left[\frac{i m_{g} c}{\hbar} \int_{\mathrm{P}}^{\mathrm{Q}} B^{a}{ }_{\mu} u_{a} d x^{\mu}\right] .
$$

Similarly to the electromagnetic phase factor, it represents the quantum mechanical law that replaces the classical gravitational Lorentz force equation (17).

\subsection{The COW Experiment}

As a first application of the gravitational nonintegrable phase factor (31), we consider the COW experiment [7]. It consists in using a neutron interferometer to observe the quantum mechanical phase shift of neutrons caused by their interaction with Earth's gravitational field, which is usually assumed to be Newtonian. Furthermore, as the experience is performed with thermal neutrons, it is possible to use the small velocity approximation. In this case, the gravitational phase factor (31) becomes

$$
\Phi_{g}(\mathrm{P} \mid \mathrm{Q})=\exp \left[\frac{i m_{g} c^{2}}{\hbar} \int_{\mathrm{P}}^{\mathrm{Q}} B_{00} d t\right],
$$

where we have used that $u^{0}=\gamma \simeq 1$ for the thermal neutrons. In the Newtonian approximation, we can set $c^{2} B_{00} \equiv \phi=g z$, with $\phi$ the (homogeneous) Earth Newtonian potential [8]. In this expression, $g$ is the gravitational acceleration, assumed not to change significantly in the region of the experience, and $z$ is the distance from Earth taken from some reference point. Consequently, the phase factor can be rewritten in the form

$$
\Phi_{g}(\mathrm{P} \mid \mathrm{Q})=\exp \left[\frac{i m_{g} g}{\hbar} \int_{\mathrm{P}}^{\mathrm{Q}} z(t) d t\right] \equiv \exp i \varphi .
$$




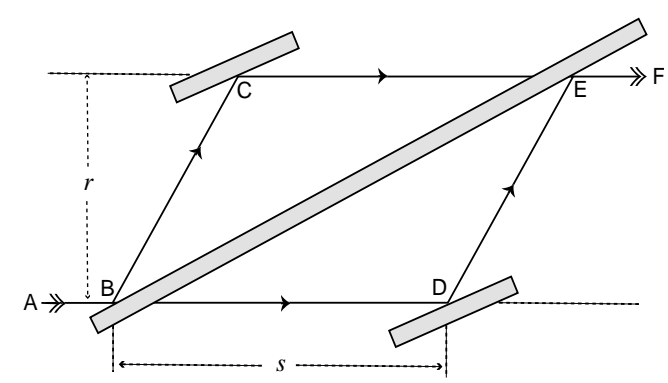

Figure 1. Schematic illustration of the COW neutron interferometer.

Let us now compute the phase $\varphi$ through the two trajectories of Fig. 1. As the phase contribution along the segments $\mathrm{DE}$ and $\mathrm{BC}$ are equal, they cancel out and do not contribute to the phase. Assuming that the segment BD is at $z=0$, we obtain for the trajectory BDE:

$$
\varphi_{\mathrm{BDE}}=\frac{m_{g} g}{\hbar} \int_{\mathrm{D}}^{\mathrm{E}} z(t) d t .
$$

For the trajectory BCE, we have

$$
\varphi_{\mathrm{BCE}}=\frac{m_{g} g}{\hbar} \int_{\mathrm{B}}^{\mathrm{C}} z(t) d t+\frac{m_{g} g r}{\hbar} \int_{\mathrm{C}}^{\mathrm{E}} d t .
$$

Therefore, we get

$$
\Delta \varphi \equiv \varphi_{\mathrm{BCE}}-\varphi_{\mathrm{BDE}}=\frac{m_{g} g r}{\hbar} \int_{\mathrm{C}}^{\mathrm{E}} d t .
$$

Since the neutron velocity is constant along the segment CE, we have

$$
\int_{\mathrm{C}}^{\mathrm{E}} d t \equiv \frac{s}{v}=\frac{s m_{i} \lambda}{h},
$$

where $s$ is the length of the segment CE, and $\lambda=h /\left(m_{i} v\right)$ is the de Broglie wavelength associated with the neutron. The gravitationally induced phase difference predicted for the COW experience is then found to be [8]

$$
\Delta \varphi=s \frac{2 \pi g r \lambda m_{i}^{2}}{h^{2}}\left(\frac{m_{g}}{m_{i}}\right) .
$$

When the gravitational and inertial masses are assumed to coincide, the phase shift becomes

$$
\Delta \varphi=s \frac{2 \pi g r \lambda m^{2}}{h^{2}}
$$

which is exactly the result obtained for the COW experiment [7].

\subsection{Gravitational Aharonov-Bohm Effect}

As a second application we use the phase factor (31) to study the gravitational analog of the Aharonov-Bohm effect [9]. The usual (electromagnetic) Aharonov-Bohm effect consists in a shift, by a constant amount, of the electron interferometry wave pattern, in a region where there is no magnetic field, but there is a nontrivial gauge potential $A_{i}$. Analogously, the gravitational Aharonov-Bohm effect will consist in a similar shift of the same wave pattern, but produced by the presence of a gravitational gauge potential $B_{0 i}$. Phenomenologically, this kind of effect might be present near a massive rapidly rotating source, like a neutron star, for example. Of course, differently from an ideal apparatus, in a real situation the gravitational field cannot be completely eliminated, and consequently the gravitational Aharonov-Bohm effect should be added to the other effects also causing a phase change.

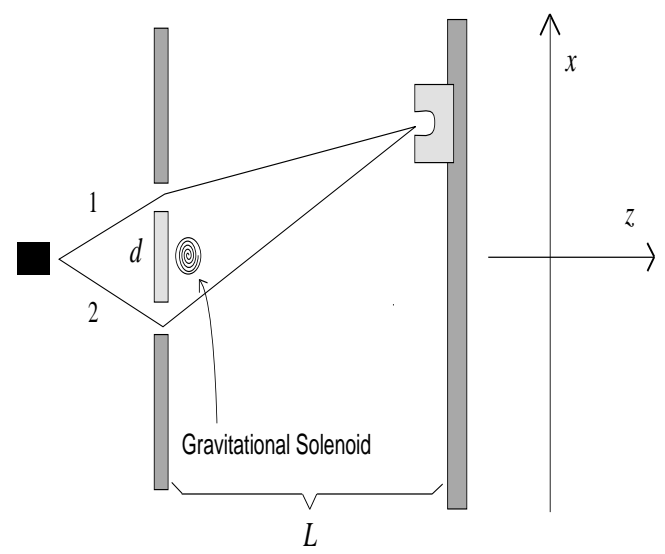

Figure 2. Schematic illustration of the gravitational AharonovBohm electron interferometer.

Let us consider first the case in which there is no external field at all. If the electrons are emitted with a characteristic momentum $p$, then its wavefunction has the de Broglie wavelength $\lambda=h / p$. Denoting by $L$ the distance between slit and screen (see Fig. 2), and by $d$ the distance between the two holes, when the conditions $L \gg \lambda, L \gg x$ and $L \gg d$ are satisfied, the phase difference at a distance $x$ from the central point of the screen is given by

$$
\delta^{0} \varphi(x)=\frac{2 \pi x d}{L \lambda} .
$$

This expression defines the wave pattern on the screen.

We consider now the ideal case in which a kind of infinite "gravitational solenoid" produces a purely static gravitomagnetic field flux concentrated in its interior. In the ideal situation, the gravitational field outside the solenoid vanishes completely, but there is a nontrivial gauge potential $B_{0 i}$. When we let the electrons to move outside the solenoid, phase factors corresponding to paths lying on one side of the solenoid will interfere with phase factors corresponding to paths lying on the other side, which will produce an additional phase shift at the screen. Let us then calculate 
this additional phase shift. The gravitational phase factor (31) for the physical situation described above is

$$
\Phi_{g}(\mathrm{P} \mid \mathrm{Q})=\exp \left[-\frac{i m_{g} c}{\hbar} \int_{\mathrm{P}}^{\mathrm{Q}} u^{0} \vec{B}_{0} \cdot d \vec{r}\right]
$$

where $\vec{B}_{0}$ is the vector with components $B_{0}{ }^{i}=-B_{0 i}$. Since $u^{0}=\gamma \equiv\left[1-\left(v^{2} / c^{2}\right)\right]^{-1 / 2}$, and considering that the electron velocity $v$ is constant, the phase difference at the screen will be

$$
\delta \varphi \equiv \varphi_{2}-\varphi_{1}=\frac{\gamma m_{g} c}{\hbar} \oint \vec{B}_{0} \cdot d \vec{r} .
$$

Since the integral

$$
\oint \vec{B}_{0} \cdot d \vec{r}=\oint\left(\vec{\nabla} \times \vec{B}_{0}\right) \cdot d \vec{\sigma}=\oint \vec{H} \cdot d \vec{\sigma} \equiv \Omega
$$

represents the flux $\Omega$ of the gravitomagnetic field $\vec{H}=$ $\vec{\nabla} \times \vec{B}_{0}$ inside the solenoid, the phase shift can be written in the form

$$
\delta \varphi=\frac{\mathcal{E} \Omega}{\hbar c}\left(\frac{m_{g}}{m_{i}}\right),
$$

where $\mathcal{E}=\gamma m_{i} c^{2}$ is the electron kinetic energy. When the gravitational and inertial masses are assumed to coincide, the phase shift becomes

$$
\delta \varphi=\frac{\mathcal{E} \Omega}{\hbar c} .
$$

Expression (45) gives the phase difference produced by the interaction of the particle's kinetic energy with a gauge potential, which gives rise to the gravitational AharonovBohm effect. As this phase difference depends on the energy, it applies equally to massive and massless particles. There is a difference, however: whereas for a massive particle it is a genuine quantum effect, for massless particles, due to the their intrinsic wave character, it can be considered as a classical effect. In fact, for $\mathcal{E}=\hbar \omega$, Eq. (45) becomes

$$
\delta \varphi=\frac{\omega \Omega}{c},
$$

and we see that, in this case, the phase difference does not depend on the Planck's constant. It is important to remark that, like the electromagnetic case, the phase difference is independent of the position $x$ on the screen, and consequently the whole wave pattern defined by (40) will be shifted by a constant amount.

\section{Final Remarks}

In Einstein's general relativity, a theory fundamentally based on the universality of free fall (or on the weak equivalence principle), geometry replaces the concept of gravitational force. This theory has been confirmed by all experimental tests at the classical level [15], but any violation of the principle would lead to its ruin. We notice in passing that the non-universality of the electromagnetic interaction is the reason why there is no geometric description, in the sense of general relativity, for electromagnetism.

On the other hand, the teleparallel equivalent of general relativity does not geometrize the interaction, but shows gravitation as a gauge force quite analogous to the Lorentz force of electrodynamics. It is able to describe the gravitational interaction in the absence of universality just as Maxwell's gauge theory is able to describe the non-universal electromagnetic interaction. In spite of the equivalence with general relativity [16], it can be considered as a more fundamental theory as it dispenses with one assumption. Notice in this connection that the equivalence principle is frequently said to preclude the definition of a local energy-momentum density for the gravitational field [17]. Although this is a true assertion in the context of general relativity, it has already been shown that a tensorial expression for the gravitational energy-momentum density is possible in the context of teleparallel gravity [11], which shows the consistency of the results.

Now, at the quantum level, deep conceptual changes occur with respect to classical gravity, the most important being the fact that gravitation seems to be no more universal [18]. In fact, at this level, the phase of the particle wavefunction acquires a fundamental status, and turns out to depend on the particle mass (in the COW effect, obtained in the non-relativistic limit), or on the relativistic kinetic energy (in the gravitational Aharonov-Bohm effect). Although in the specific case of the COW experiment the phase shift can be made independent of the mass by introducing a kind of quantum equivalence principle [19], the basic difficulty remains that different versions of this quantum principle would be necessary for different phenomena. Since teleparallel gravity is able to describe gravitation independently of the validity or not of the equivalence principle [5], it will not require a quantum version of this principle to deal with gravitationally induced quantum effects, and can be considered as providing a much more appropriate and consistent approach to study such effects.

Relying on the above arguments, we can say that the fundamental field describing gravitation is neither the tetrad nor the metric, but the translational gauge potential $B^{a}{ }_{\mu}$. Metric is no more a fundamental, but a derived quantity. This point can have important consequences for both classical and quantum gravity. Gravitational waves should be seen as $B$ waves and not as metric waves. Quantization of the gravitational field should be carried out on $B^{a}{ }_{\mu}$ and not on the metric. Another consequence refers to a fundamental problem of quantum gravity, namely, the conceptual difficulty of reconciling local general relativity with non-local quantum mechanics, or of reconciling the local character of the equivalence principle with the non-local character of the uncertainty principle [20]. As teleparallel gravity can be formulated independently of the equivalence principle, the quantization of the gravitational field may possibly appear more consistent if considered in the teleparallel picture.

\section{Acknowledgments}

The authors thank J. M. Nester for useful discussions. They also thank FAPESP-Brazil, CAPES-Brazil, and CNPq-Brazil for partial financial support. 


\section{References}

[1] For a general description of the gauge approach to gravitation, see F. W. Hehl, J. D. McCrea, E. W. Mielke, and Y. Ne'emann, Phys. Rep. 258, 1 (1995); see also M. Blagojević, Gravitation and Gauge Symmetries (IOP Publishing, Bristol, 2002).

[2] The basic references on torsion gravity can be found in R. T. Hammond, Rep. Prog. Phys. 65, 599 (2002).

[3] The name teleparallel gravity is normally used to denote the general three-parameter theory introduced in K. Hayashi and T. Shirafuji, Phys. Rev. D19, 3524 (1979). Here, however, we use it as a synonymous for the teleparallel equivalent of general relativity, a theory obtained for a specific choice of these parameters. For a recent appraisal on the three-parameter teleparallel gravity, see Yu. N. Obukhov and J. G. Pereira, Phys. Rev. D67, 044016 (2003).

[4] V. C. de Andrade and J. G. Pereira, Phys. Rev. D56, 4689 (1997).

[5] R. Aldrovandi, J. G. Pereira, and K. H. Vu, Gen. Rel. Grav. 36, 101 (2004) [gr-qc/0304106].

[6] T. T. Wu and C. N. Yang, Phys. Rev. D12, 3845 (1975).

[7] A. W. Overhauser and R. Colella, Phys. Rev. Lett. 33, 1237 (1974); R. Colella, A. W. Overhauser, and S. A. Werner, Phys. Rev. Lett. 34, 1472 (1975).

[8] R. Aldrovandi, J. G. Pereira, and K. H. Vu, Class. Quant. Grav. 21, 51 (2004) [gr-qc/0310110].

[9] Different phenomena have been named "gravitational Aharonov-Bohm effect" in the literature. See, for example, P. O. Mazur, Phys. Rev. Lett. 57, 929 (1986); P. O. Mazur, Phys. Rev. Lett. 59, 2380 (1987); A. K. Lawrence, D. Leiter, and G. Samozi, Nuovo Cimento 17B, 113 (1973); L. H. Ford and A. Vilenkin, J. Phys. A14, 2353 (1981); V. B. Bezerra, J. Math. Phys. 30, 2895 (1989); V. B. Bezerra, Class. Quant. Grav. 8, 1939 (1991).
[10] See, for example, R. Aldrovandi and J. G. Pereira, An Introduction to Geometrical Physics (World Scientific, Singapore, 1995).

[11] V. C. de Andrade, L. C. T. Guillen, and J. G. Pereira, Phys. Rev. Lett. 84, 4533 (2000).

[12] See the Preface of J. L. Synge, Relativity: The General Theory (J. Wiley, New York, 1960); see also T. Damour, Contribution to the ONERA workshop on "Missions spatiales en physique fondamentale", to appear in a special issue of the Comptes Rendus de l'Académie des Sciences (Paris), 2001, ed. C. Bordé and P. Touboul [gr-qc/0109063].

[13] L. D. Landau and E. M. Lifshitz, The Classical Theory of Fields (Pergamon, Oxford, 1975).

[14] Y. Aharonov and D. Bohm, Phys. Rev. 115, 485 (1959).

[15] For a recent overview on the experimental tests of the equivalence principle, see C. M. Will, Living Rev. Rel. 4, 4 (2001); see also M. P. Haugan and C. Lämerzahl, Lect. Notes Phys. 562, 195 (2001).

[16] This is true even in the presence of spinor fields, provided the appropriate spin connection be used; see V. C. de Andrade, L. C. T. Guillen, and J. G. Pereira, Phys. Rev. D64, 027502 (2001).

[17] C. W. Misner, K. S. Thorne, and J. A. Wheeler, Gravitation (Freeman, New York, 1973).

[18] D. Greenberger, Ann. Phys. (NY) 47, 116 (1968); D. Greenberger and A. W. Overhauser, Rev. Mod. Phys. 51, 43 (1979).

[19] C. Lämmerzahl, Gen. Rel. Grav. 28, 1043 (1996); C. Lämmerzahl, Acta Phys. Pol. 29, 1057 (1998).

[20] C. Lämmerzahl, Gen. Rel. Grav. 28, 1043 (1996); C. Lämmerzahl, Acta Phys. Polon. 29, 1057 (1998); R. Y. Chiao, in Wheeler's 90th Birthday Symposium Proceedings (Cambridge University Press, Cambridge, 2003) [gr-qc/0303100]. 